\title{
Efficiency Based Design Flow For Fully-Integrated Class C RF Power Amplifiers In Nanometric CMOS
}

\author{
Nicolás Barabino \\ Instituto de Ingeniería Eléctrica \\ Universidad de la República \\ Montevideo, Uruguay \\ barabino@fing.edu.uy
}

\author{
Rafaella Fiorelli \\ IMSE CNM-CSIC \\ Universidad de Sevilla \\ Seville, Spain \\ fiorelli@imse-cnm.csic.es
}

\author{
Fernando Silveira \\ Instituto de Ingeniería Eléctrica \\ Universidad de la República \\ Montevideo, Uruguay \\ silveira@fing.edu.uy
}

\begin{abstract}
A design flow for class C radiofrequency (RF) power amplifiers (PAs) with on-chip passive output network in nanometric technologies is proposed. While class C PAs achieve high efficiency with off-chip output networks when fullyintegrated, low-Q discrete components decrease the efficiency and present a trade-off in the design. This work intends to bring a solution to that trade-off. The design flow is based in actual transistors' DC curves and inductors simulation data provided by the foundry. A complete design space map of efficiency and components design for a given power and harmonic level at the output is generated, allowing the designer to select the best tradeoff. A design example for a IEEE 802.15.4 2.4 GHz compliant $P A$ in a $90 \mathrm{~nm}$ CMOS technology is presented.
\end{abstract}

\section{INTRODUCTION}

The notorious increment of very low power wireless applications using on-chip transceivers, force Power Amplifier (PA) designers to reduce the consumption of this power hungry block as much as possible without jeopardizing its performance. The use of nanometric technologies, whereas makes possible RF low power design, requires to handle the non-ideal characteristics of transistors and low supply voltages. Furthermore, full integration using on-chip, low quality factor inductors, also presents a challenge. This paper presents a design approach that handles these aspects: allow the designer to select the most appropriate trade-off between efficiency and components size for a given output power and harmonic content, while taking into account the actual transistor and inductor characteristics.

In constant modulation schemes where linearity is not needed in the output stage, as in the IEEE 802.15.4 standard, high efficiencies can be achieved due to the use of nonlinear PAs with off-chip output networks (for example class $\mathrm{C}$ or $\mathrm{E})$. However, the use of fully integrated output network adds considerable losses that do not allow the implementation of highly efficient PAs and also makes the definition of the optimum design a challenging task. Some works have proposed parasitic-aware design flows [?], [?] based on a simulator on the loop approach. It is the aim of this work to present a solution with a simple parasitic-aware "feedforward" approach, for a particular case of the class C PA, though the general approach can be applied to other topologies. This "feed-forward" approach provides a global view of the trade-offs and a way to select an initial design.
In order to solve the design's trade offs, the transistor's input voltage space (represented by the DC and RF signal components) is swept using Matlab routines and a design map is generated, allowing the designer to select the best configuration, e.g. with maximum efficiency. To do so in each design point the transistor's behavior is analyzed and an optimum output network and transistor size are designed. The input network is not considered in the analysis and the efficiency studied is the one of the power supply to RF conversion.

Traditional theoretical analysis of class C PAs efficiency considers a fairly ideal transistor [?], [?], [?]. Despite this simple approach is useful for long channel transistors, it is not the case when nanometric devices are used, e.g. because of its drain current strong dependency with drain voltage variations. So an approach was developed, which is new as far as the authors know, which is based on actual DC drain current vs. gate and drain voltages curves. This enables to take into account second order and short channel effects, as well as sub-threshold operation. Parasitic capacitances of the transistor are not considered in order to simplify the analysis, as their impact is not important due to the technology used and the transistor dimensions considered when focusing in low power applications, e.g. IEEE 802.15.4 standard.

To study the efficiency of the output network the parasitics of the on-chip inductors were reckoned in our routines by acquiring inductor characteristics through simulation using the information provided in the foundry inductor library.

This work is laid out as follows. Section ?? introduces the PA architecture used in this work. In Section ?? the proposed design flow is described. Finally, Section ?? shows a particular PA design following the procedure discussed previously.

\section{PA ARCHITECTURE}

The schematic shown in Fig. ?? is composed by a MOS transistor and an arbitrary output network, it could represent a class $\mathrm{A}, \mathrm{AB}, \mathrm{B}$ or $\mathrm{C} \mathrm{PA}$ [?]. The operating class or conduction angle (fraction of the period where the transistor conducts) is to be determined by the gate's bias $v_{G}^{D C}$ and signal amplitude $v_{G}^{R F}$. Then, the expression of $v_{G}$ is

$$
v_{G}=v_{G}^{D C}+v_{G}^{R F} \sin \left(\omega_{0} t\right)
$$




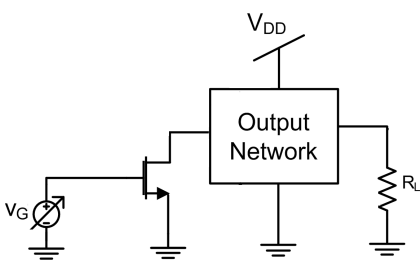

(a) PA Architecture

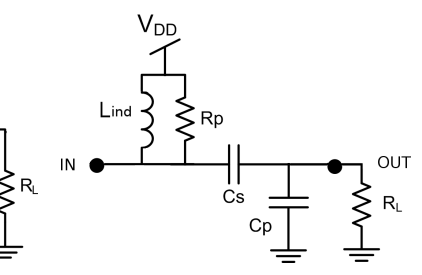

(b) Proposed output network
The output network is utilized: (i) to bias the drain to the supply voltage $V_{D D}$; (ii) to present a properly designed impedance value $R_{O}$ at the working frequency to obtain the desired output power; and (iii) to filter the harmonic currents. The amount of filtering depends on the desired application. Considering the IEEE 802.15.4 standard, harmonics power must be $20 \mathrm{~dB}$ below the fundamental output.

Assuming the harmonics filtered, the drain voltage is approximately sinusoidal

$$
\begin{gathered}
v_{D}=V_{D D}-v_{D}^{R F} \sin \left(\omega_{0} t\right) \\
v_{D}^{R F}=R_{O} I_{\text {fund }}
\end{gathered}
$$

with $I_{f u n d}$ the amplitude of the Fourier fundamental component of the drain current.

The MOS drain efficiency $\eta_{M O S}$ (RF power delivered to the output network over DC power taken from $V_{D D}$ ) and the network efficiency $\eta_{N W}$ (power delivered to the load over power taken from the transistor) are

$$
\begin{gathered}
\eta_{M O S}=\frac{P_{N W}}{P_{D C}}=\frac{I_{\text {fund }} \times v_{D}^{R F}}{2 I_{D C} V_{D D}} \\
\eta_{N W}=\frac{P_{R L}}{P_{N W}}
\end{gathered}
$$

So the total PA efficiency $\eta$ can be written as

$$
\eta=\eta_{M O S} \times \eta_{N W}
$$

The drain voltage amplitude $v_{D}^{R F}$ must be close to $V_{D D}$ in order to have high $\eta_{M O S}$ according to (??). So the maximum $\left(v_{D, \max }\right)$ and minimum $\left(v_{D, \text { min }}\right)$ for the drain voltage swing have to be determined by the maximum drain voltage the technology can support $(1.2 \mathrm{~V}$ for standard $90 \mathrm{~nm}$ MOS transistors in our case) and by the minimum so that the drain current is not cut. There is some freedom in choosing this one, it was found $0.2 \mathrm{~V}$ to be a good choice for the studied technology. Then $v_{D}^{R F}$ and $V_{D D}$ are determined by

$$
\begin{aligned}
v_{D}^{R F} & =\frac{v_{D, \max }-v_{D, \min }}{2} \\
V_{D D} & =\frac{v_{D, \max }+v_{D, \min }}{2}
\end{aligned}
$$

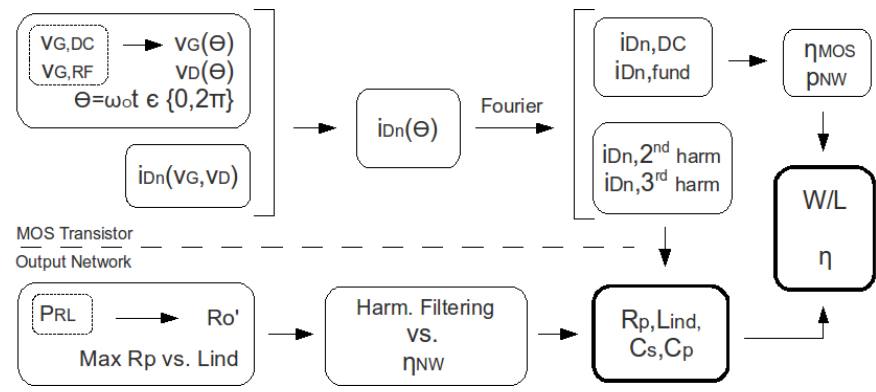

Figure 1. Flow diagram of the method proposed.

\section{PA DESIGN FLOW}

To generate the efficiency design map the gate voltage parameters $v_{G}^{D C}$ and $v_{G}^{R F}$ are swept. The portion of the space explored is $v_{G}^{D C}+v_{G}^{R F}<v_{G, \text { max }}$. For the technology being used $v_{G, \max }$ is $1 \mathrm{~V}$.

In each point, for a given output power $\left(P_{R L}\right)$, the transistor's size $(W / L)$ and efficiency $\left(\eta_{M O S}\right)$ are calculated, an optimum output network is designed (intended to maximize $\left.\eta_{N W}\right)$ and the PA's total efficiency $(\eta)$ computed.

This procedure is separated in two analysis, the first only considers the behavior of the transistor and the second one designs the lumped network. The first analysis is independent of which output network is used. Whereas the second is done using a particular network topology. At the end of the procedure both analysis are combined to obtain the global result of efficiency and components design.

The complete analysis flow for each design point, which was implemented in Matlab routines, is shown in Fig.??. The next sub-sections describe each step.

\section{A. MOS Transistor analysis}

As it was already explained, in order to model the transistor's drain current accurately its DC value is extracted from simulation (in SpectreRF with BSIM4 for the selected $90 \mathrm{~nm}$ technology) varying both $v_{G}$ and $v_{D}$. Then $i_{D}$ data is normalized regarding transistor width and length ratio $W / L$, obtaining $i_{D n}\left(v_{G}, v_{D}\right)$. Having the channel length $L$ fixed, these curves are slightly dependent on $W$, so the normalization is only valid for certain range. This is solved having independent data for a few $W$ regions.

Using the $i_{D n}\left(v_{G}, v_{D}\right)$ data the time domain normalized drain current $i_{D n}(\theta)$ (with $\theta \in\{0,2 \pi\}$ ) is calculated starting from $v_{G}$ and $v_{D}$ time domain voltages, from (??) and (??). Then, its first four Fourier terms are calculated $\left(i_{D n, D C}\right.$, $i_{D n, f u n d}, i_{D n, 2^{\text {nd }}}$ harm and $\left.i_{D n, 3^{\text {rd }} \text { harm }}\right)$. With the normalized current the conduction angle, $\theta_{\text {cond }}$ which indicates the operating class and the amount of non-linearity, can also be obtained

Then, from (??), $\eta_{M O S}$ is calculated. Also $p_{N W}$, the power delivered to the output network normalized by $W / L$ is computed using (??).

$$
p_{N W}=\frac{i_{D n, f u n d} \times v_{D}^{R F}}{2}
$$




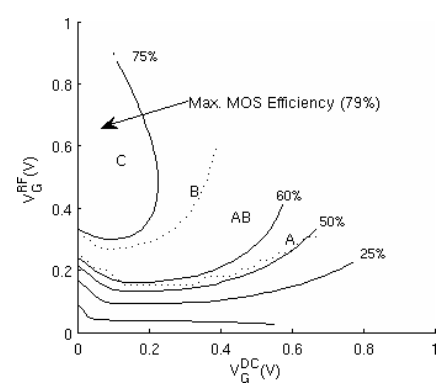

(a) MOS Efficiency

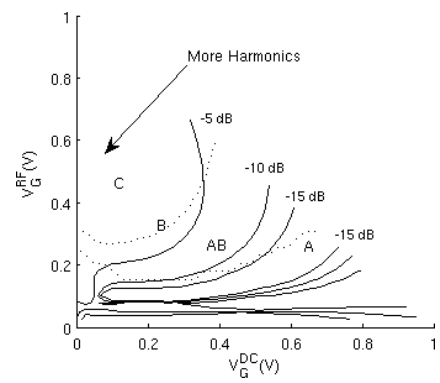

(c) Second harmonic

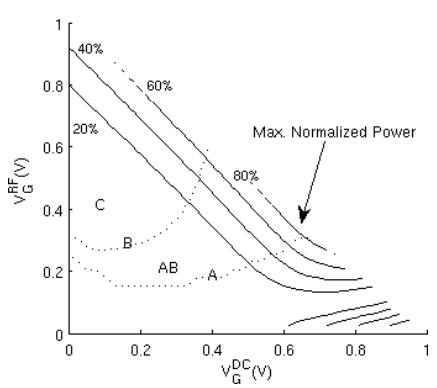

(b) Normalized Power $\left(\frac{p_{\mathrm{NW}}}{\max \left(p_{\mathrm{NW}}\right)}\right)$

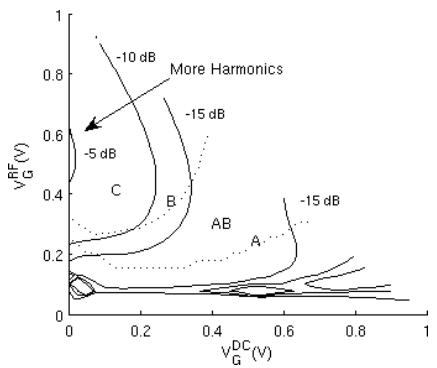

(d) Third harmonic
Figure 2. MOS Efficiency, Normalized power and harmonics in the design space

Figs. ?? and ?? show efficiency and normalized power contours versus pair $\left(v_{G}^{D C}, v_{G}^{R F}\right)$. PA class divisions are also shown according to conduction angle ${ }^{1}$. As expected, when moving from class $\mathrm{A}$ to class $\mathrm{C} \eta_{M O S}$ rises (the maximum is obtained with zero bias), while normalized power drops. In Figs. ?? and ??, second and third harmonics contours of $i_{D n}$ are shown; and as expected harmonics rise when moving from class A to class $\mathrm{C}$.

\section{B. Output network analysis}

In this work we choose to use a fully integrated $\pi$ network, as depicted in Fig. ??. Similar topologies can be found in other class C PA implementations, e.g. [?]. This network can comply with the requirements for the output network described in section ??, but with the restriction that $R_{O}$ must be greater than $R_{L}$. This is not an inconvenient when low power outputs are needed (e.g. in IEEE 802.15.4), where $R_{O}$ values are always higher than $R_{L}$ (assumed to be $50 \Omega$ ). It has only one degree of freedom, for example having chosen a value for $L_{\text {ind }}$ the values for $C_{s}$ and $C_{p}$ are exactly determined. This network was chosen for its simplicity and for using only one inductor, because inductors are generally the main cause for network losses. The inductor losses, represented by its parallel resistance $R_{p}$, determines the efficiency. The network's capacitors are supposed lossless.

The resistance $R_{O}$ shown by the network at the fundamental frequency is the parallel of $R_{p}$ and $R_{O}{ }^{\prime}$, where $R_{O}{ }^{\prime}$ is the resistance converted from $R_{L}$ at the fundamental frequency

\footnotetext{
${ }^{1}$ Here we calculate the conduction angle as the portion of the period while the drain current is higher than the $1 \%$ of its maximum.
}

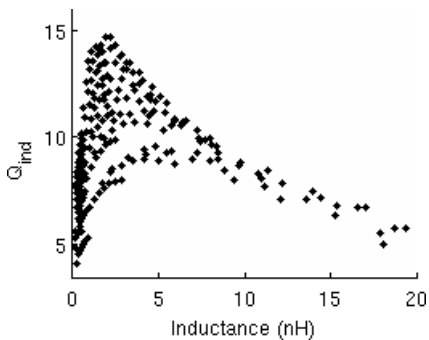

(a) Quality factor

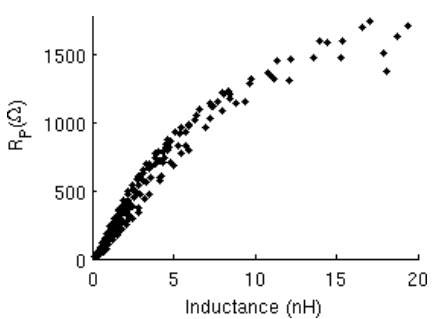

(b) Parallel resistance
Figure 3. $Q_{\text {ind }}$ and $R_{p}$ vs. $L_{\text {ind }}$

by the $\pi$ network $\left(L_{\text {ind }}, C_{s}\right.$ and $C_{p}$ ) without considering the inductor losses. The power delivered to the load $P_{R L}$ is the power taken by $R_{O}{ }^{\prime}$, so $P_{R L}$ and the efficiency of the network $\eta_{N W}$ (??) can be written as

$$
\begin{gathered}
P_{R L}=\frac{v_{D}^{R F^{2}}}{2 R_{O}{ }^{\prime}} \\
\eta_{N W}=\frac{R_{p}}{R_{p}+R_{O}{ }^{\prime}}
\end{gathered}
$$

Hence, a large value of parallel parasitic resistance $R_{p}$ is required to achieve high efficiency. This parasitic resistance is related to the inductor's quality factor as $R_{p}=Q_{\text {ind }} \times\left(\omega_{0} L_{\text {ind }}\right)$.

For the technology used in this work, we swept the inductor design space in order to find the maximum quality factor available vs. the inductance value, as well as the geometry of those inductors. Fig. ?? shows the $Q_{\text {ind }}$ and $R_{p}$ values (at $2.4 \mathrm{GHz}$, the working frequency in our case) vs. inductance $L_{\text {ind }}$. The inductors that have the maximum parallel resistance for each inductance value are the ones used in the network design routines.

The quality factor of the network -and so the network's filtering capability- decreases when $L_{\text {ind }}$ increases, while $R_{p}$ augments, as it is seen in Fig. ??. Therefore, to have the best network efficiency the inductance $L_{\text {ind }}$ must be chosen as high as possible while the network sufficiently filters the harmonics.

Given the required output power $P_{R L}$ a network is designed (i.e. the capacitors' values) for each inductor, in order to show the correct $R_{O}{ }^{\prime}$ value (obtained by using (??)). Then its amount of harmonic filtering is calculated. An example of filtering vs. inductance is depicted in Fig. ??. The amount of filtering is calculated for the second and third harmonics (supposing the higher ones are negligible), although as shown in Figs. ?? and ?? the second is almost always stronger.

\section{Total efficiency and components design}

With the harmonics values $\left(i_{D n, 2^{\text {nd }} \text { harm }}\right.$ and $\left.i_{D n, 3^{\text {rd }} \text { harm }}\right)$ and the harmonics filtering vs. network design, the optimum network (the one that achieves the needed output filtering with highest efficiency) is chosen for each design space point.

Fig. ?? shows an example of the network efficiency $\eta_{N W}$ contours . As it is expected, the network efficiency decreases when moving from Class $\mathrm{A}$ to Class $\mathrm{C}$ zone, due to higher filtering requirements. 


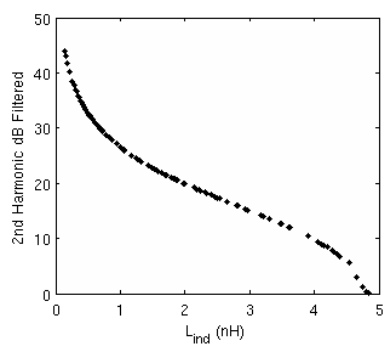

(a) Second harmonic filtering Vs. $L_{\text {ind }}$

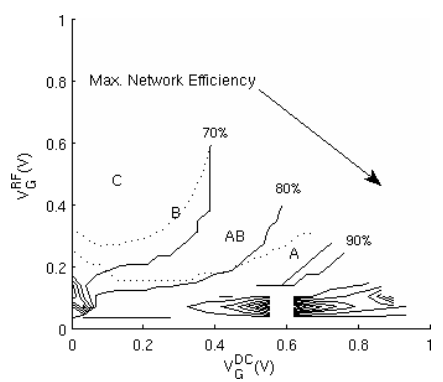

(b) Network efficiency $\eta_{N W}$
Figure 4. Harmonic filtering Vs. $L_{\text {ind }}$ and network efficiency contour

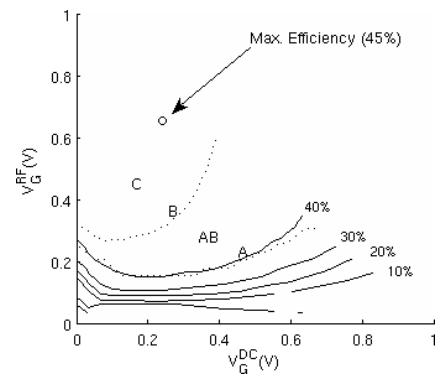

(a) PA total efficiency

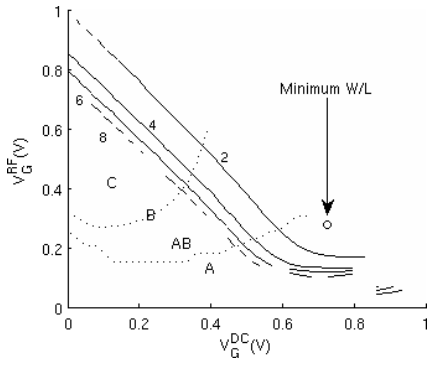

(b) Transistor's W/L (normalized to its minimum)
Figure 5. PA total efficiency and transistor's W/L contours

Then, combining normalized power delivered to the network $p_{N W}$ (??) and network efficiency $\eta_{N W}$ (??) the transistor's aspect ratio is calculated as

$$
W / L=\frac{P_{R L}}{\eta_{N W} p_{N W}}
$$

Also total efficiency $\eta$ is calculated with (??).

The contours of efficiency $\eta$, transistor's aspect ratio $W / L$ (both shown in Fig. ??) and the ones of inductance and capacitances values form a design space map which can be used by the designer to select an optimum design. For example it could be found the design with the highest efficiency given certain constraints for the components sizes. It is to be noted that this optimum can be in any of the classes regions $(\mathrm{A}, \mathrm{AB}, \mathrm{B}, \mathrm{C})$ and not only in class $\mathrm{C}$.

\section{Design EXAMPle}

This section presents a proof of concept regarding the design method here proposed. We studied the design space for PA compliant with IEEE 802.15.4 2.4 GHz standard given two

\begin{tabular}{|c|c|c|c|c|c|c|c|c|c|c|}
\hline \multirow{2}{*}{$\begin{array}{l}v_{G}^{\mathrm{DC}} \\
(\mathrm{V})\end{array}$} & \multirow{2}{*}{$\begin{array}{l}v_{G}^{\mathrm{RF}} \\
(\mathrm{V})\end{array}$} & \multirow{2}{*}{$\begin{array}{l}W \\
(\mu \mathrm{m}\end{array}$} & \multirow{2}{*}{$\begin{array}{l}L_{\text {ind }} \\
(\mathrm{nH})\end{array}$} & \multirow{2}{*}{$\begin{array}{l}C_{s} \\
(\mathrm{pF})\end{array}$} & \multirow{2}{*}{$\begin{array}{l}C_{p} \\
(\mathrm{pF})\end{array}$} & \multicolumn{2}{|c|}{$P_{R L}(\mathrm{dBm})$} & \multicolumn{2}{|c|}{$\eta(\%)$} & $\begin{array}{l}2^{\text {nd }} \text { Harm } \\
(\mathrm{dB})\end{array}$ \\
\hline & & & & & & Pred. & Sim. & Pred & Sim & Sim. \\
\hline 0.4 & 0.5 & 76 & 1.7 & 6.7 & 3.8 & 0 & -0.1 & 42.7 & 41.5 & -20.3 \\
\hline 0.2 & 0.8 & 23 & 4.0 & 1.6 & 2.7 & -5 & -5.1 & 41.9 & 40.1 & -21.2 \\
\hline
\end{tabular}

Table I

PREDICTED VS SIMULATED RESULTS

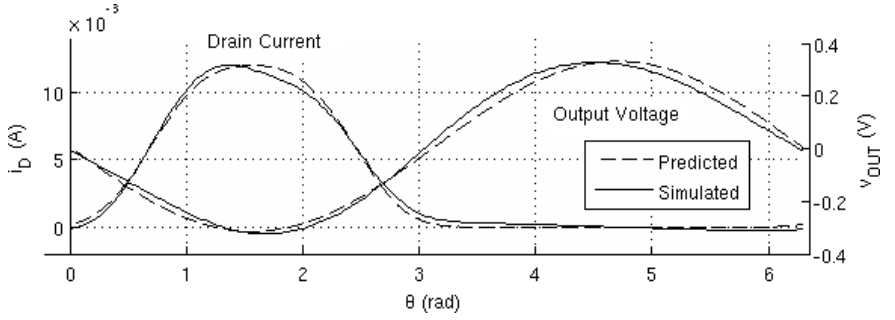

Figure 6. Comparison between Matlab and SpectreRF (pss) time-domain results

output power requirements $(0 \mathrm{dBm}$ and $-5 \mathrm{dBm})$. The highest efficiency configurations were selected and the predicted performances were compared with simulations carried out in SpectreRF using the periodic steady state analysis.

The transistors used were $1.2 \mathrm{~V}$ standard NFET with minimal channel length $(100 \mathrm{~nm})$. These low voltage transistors were found to be suitable due to the low output power required. The load was set to $R_{L}=50 \Omega$ and to maximize the drain swing (as explained in Section ??) $V_{D D}$ was chosen to be 0.7 V.

Table ?? presents the designs and their predicted and simulated performance. In Fig. ?? the waveforms of drain current and output voltage are given for the $0 \mathrm{dBm}$ case.

Both performance and time-domain comparisons show good agreement between predicted and simulated results.

\section{CONCLUSION}

A design flow intended to find highest efficiency configurations for Class C RF Power Amplifiers is proposed. This method provides the designer a design space map of efficiency and components (transistors, inductors, capacitors) parameters starting from output requirements (load, power, harmonics) and actual transistors and inductors data.

The method is tested by comparing its predicted results with simulations carried out in SpectreRF for a $90 \mathrm{~nm}$ technology for a IEEE 802.15.4, $2.4 \mathrm{GHz}$ compliant, design example.

\section{ACKNOWLEDGEMENTS}

The authors would like to thank the support of grant ANII BE_POS_1250, Uruguayan projects PDT 69/08 and FCE_2007_501; and Catrene European project 2A105SR2 and Avanza I+D Spanish project TSI-020400-2008-71.

\section{REFERENCES}

[1] R. Gupta, B. M. Ballweber, and D. J. Allstot, "Design and optimization of CMOS RF power amplifiers," IEEE Journal of Solid-State Circuits, vol. 36, no. 2, pp. 166-175, Feb. 2001.

[2] K. Choi and D. J. Allstot, "Parasitic-aware design and optimization of a CMOS Rf power amplifier," IEEE Transactions on Circuits and SystemsPart I: Fundamental Theory and Applications, vol. 53, no. 1, pp. 16-25, Jan. 2006.

[3] T. H. Lee, The design of CMOS radio-frequency integrated circuits, 2nd ed. Cambridge University Press, 2004.

[4] B. Razavi, $R F$ Microelectronics, 1st ed. Prentice Hall, 1998.

[5] D. Zito, D. Pepe, and B. Neri, "Low-power rf transceiver for ieee 802.15.4 (zigbee) standard applications," in Electronics, Circuits and Systems, 2006. ICECS '06. 13th IEEE International Conference on, Dec. 2006, pp. 1312-1315. 